\title{
Thickness effects on fibre-bridged fatigue delamination growth in composites
}

\author{
Liaojun Yao ${ }^{\mathrm{a},{ }^{*}}$, Hao Cui ${ }^{\mathrm{b}}$, R.C. Alderliesten ${ }^{\mathrm{c}}$, Yi Sun ${ }^{\mathrm{a}}$, Licheng Guo $^{\mathrm{a}}$ \\ ${ }^{a}$ Department of Astronautics Science and Mechanics, Harbin Institute of Technology, \\ Harbin, P.R. China \\ ${ }^{\mathrm{b}}$ School of Aerospace, Transport and Manufacturing, Cranfield University, United
} Kingdom

${ }^{\mathrm{c}}$ Structural Integrity and Composites Group, Faculty of Aerospace Engineering, Delft University of Technology, The Netherlands

* Corresponding author email: $\underline{\text { L.Yao@hit.edu.cn }}$

\section{ABSTRACT:}

This paper provides an investigation on thickness effects on fibre-bridged fatigue delamination growth (FDG) in composite laminates. A modified Paris relation was employed to interpret experimental fatigue data. The results clearly demonstrated that both thickness and fibre bridging had negligible effects on FDG behaviors. Both energy principles and fractography analysis were subsequently performed to explore the physical reasons of this independence. It was found that the amount of energy release of a given crack growth was not only independent of fibre bridging, but also thickness. Fibre print was the dominant microscopic feature located on fracture surfaces, physically making the same energy dissipation during FDG. Furthermore, the present study provides extra evidence on the importance of using an appropriate similitude parameter in FDG studies. Particularly, the strain energy release rate (SERR) range applied around crack front was demonstrated as an appropriate similitude 
parameter for fibre-bridged FDG study.

Keywords: B. Fatigue; B. Delamination; Fibre bridging; A. Polymer-matrix composites (PMCs)

\section{Introduction}

Composite laminates, with widespread use in high-tech industry, are vulnerable to delamination growth, owing to lack of reinforcement in thickness direction. This damage can gradually propagate under cyclic loading and may finally result in catastrophic failure of a composite structure during its service life. In the past, engineers usually applied no crack growth philosophy in composite structural design, which can significantly reduce weight-saving potential of composites. Since 2009, the US Federal Aviation Administration (FAA) has changed the design philosophy of composite structures from no crack growth to slow crack growth in the certification procedure [1]. This change makes it even crucial to have in-depth understanding of FDG in composites.

People indeed have paid a lot of attention into FDG, and as a result, a vast number of research papers have been published on this topic [2-8]. Pascoe et al [5] gave a critical literature review on FDG studies in both composites and adhesive bonds. It was reported that methods based on the fracture mechanics were useful to determine FDG behaviors. Particularly, the Paris relation and its variations have been successfully employed in FDG studies. In these relations, fatigue crack growth rate $d a / d N$ was usually correlated to the SERR. However, one should bear in mind that there was no consensus on the specific expressions of SERR in these relations. Researchers usually 
would like to use the maximum SERR [2,3,4], SERR range [2,6], or combinations of them $[2,3,7,8]$ as similitude parameter to interpret FDG behaviors. The selection of an appropriate similitude parameter was one of the most important issues in FDG studies [5]. The lack of consensus on this parameter can cause controversy in fatigue data interpretation, taking stress ratio effects as examples [2-6].

Fibre bridging is a unique and important shielding mechanism frequently observed in delamination of composite laminates. The presence of bridging fibres in the wake of a crack front can bridge fracture surfaces and prohibit crack growth. Significant studies have been conducted on quasi-static delamination [9-11]. As a result, the corresponding experiment and prediction methods have been developed to characterize this shielding phenomenon. However, to the best knowledge of the authors, not enough attention has ever been paid into FDG with fibre bridging.

Hojo et al [12] proposed a $G_{\max }$-constant test program to evaluate FDG behaviors with fibre bridging. Hwang et al [13] completed FDG tests with width tapered DCB specimens under constant SERR range. Both of them found that the presence of fibre bridging can significantly retard fatigue crack growth rate. Khan et al [10] made a comparison on FDG with and without fibre bridging by removing part of fibre bridging via a thread cutting method. The results also demonstrated that fibre bridging can decrease fatigue crack growth. Yao et al [14-16] experimentally examined FDG with different amounts of fibre bridging and proposed empirical power law relations to determine fibre-bridged FDG behaviors. Furthermore, it was reported that the significance of fibre bridging was related to loading regime. Similar conclusion was 
also made by Stutz et al [17], in which bridging stress distribution in quasi-static delamination was much higher than that in fatigue. As a result, one can reason that the resistance curve (R-curve) obtained in quasi-static delamination cannot well represent resistance increase in fatigue delamination. In addition, it has been proven that stress ratio also affected the significance of fibre bridging, due to crack closure or other unknown reasons [16,18]. Particularly, fibre bridging of a high stress ratio delamination was much more significant than that of a low stress ratio.

To take fibre bridging into account, people usually applied the normalized SERR range, i.e. $\Delta G / G_{I C}\left(a-a_{0}\right)$, or the normalized maximum $S E R R$, i.e. $G_{\max } / G_{I C}\left(a-a_{0}\right)$, as similitude parameter in FDG studies $[3,7,13,19]$. Particularly, in the studies of Murri et al [3] and Hwang et al [13], they used quasi-static $R$-curve to normalize $G_{\max }$ in fatigue data analysis. However, it is questionable to directly use quasi-static data in FDG studies, as there was difference in the amount of fibre bridging generated in quasi-static and fatigue loading [14,16,17]. To address this problem, in the studies of Zhao et al $[7,19]$, the resistance increase in fatigue delamination was determined via a compliance method and subsequently used in FDG study. Once the normalized parameter was employed, fatigue data scatter can be reduced significantly and clear trend of FDG can be observed.

According to quasi-static studies $[9,20,21]$, thickness had important effects on the significance of fibre bridging. However, there was no agreement on this dependence. Some researchers found that the increase of thickness can cause more fibre bridging [21], whereas other studies provided evidence that thickness had no obvious effect on 
fibre bridging $[9,20]$. At this point, it is reasonable to ask a question that what thickness effects on fibre-bridged FDG in composite laminates. The aim of present study is, therefore, to explore fibre-bridged FDG behaviors in composite laminates with different thicknesses.

\section{Material and fatigue experimental program}

To investigate thickness effects on fibre-bridged FDG behaviors, mode I delamination tests were conducted on unidirectional DCB specimens with three thicknesses, i.e. $h=3.75 \mathrm{~mm}, h=5.0 \mathrm{~mm}$ and $h=7.5 \mathrm{~mm}$, at the same stress ratio $R=0.5$.

\subsection{Material and specimen preparation}

Composite laminates were produced by hand-lay-up of thermosetting unidirectional carbon/epoxy prepreg layers of M30SC/DT120 (high strength and modulus carbon fibre/toughened thermosetting epoxy), supplied by Delta-Tech S.p.A Italy. A $12.7 \mu \mathrm{m}$ Teflon film was inserted in the middle plane of these laminates during the hand-lay-up process to act as an initial delamination $a_{0}=60 \mathrm{~mm}$. Three laminates with different nominal thicknesses of $3.75 \mathrm{~mm}, 5.0 \mathrm{~mm}$ and $7.5 \mathrm{~mm}$ were prepared, such to investigate the influence of laminate thickness on the fatigue crack growth performance. The laminates were cured in vacuum in an autoclave at a pressure of 6 bars and curing temperature of $120^{\circ} \mathrm{C}$ for $90 \mathrm{~min}$. All laminates were $\mathrm{C}$-scanned to detect potential imperfections. These plates were subsequently cut by a diamond saw into $25 \mathrm{~mm}$ width beams with $200 \mathrm{~mm}$ length. Only these samples were tested where the C-scan did not reveal any obvious imperfections. A pair of aluminum loading blocks, $25 \mathrm{~mm}$ width by $20 \mathrm{~mm}$ length with $6 \mathrm{~mm}$ thickness, was adhesively bonded onto a specimen at the side of the Teflon insert for load introduction. 
One side of a DCB specimen was coated with thin typewriter correction fluid to enhance visibility of crack front during fatigue delamination test. A strip of grid paper was pasted on the coated side of a specimen to aid in measuring crack propagation length.

\subsection{Fatigue delamination program}

One should note that there was no test standard for mode I FDG in composites until now [4]. In practice, fatigue tests can be conducted under either displacement control or force control. In a recent study [22], it was reported that displacement control was more stable and can result in less data scatter, in comparison with force control. Thus, displacement control was applied in present study. Particularly, all fatigue tests were performed on a $10 \mathrm{kN}$ MTS machine at a frequency of $5 \mathrm{~Hz}$ with the same stress ratio $R=0.5$ in ambient conditions. A computer controlled digital camera system was employed to automatically monitor crack growth at the maximum displacement with pre-defined intervals. The force, displacement and number of cycles were stored in an Excel file every 100 cycles enabling data evaluation after the test. The experimental setup is demonstrated in Fig.1.

The amount of fibre bridging can increase with crack propagation until a full damage process zone was formed $[9,10]$. To determine FDG with different amounts of fibre bridging, DCB specimens were tested for several times with different applied displacements, but keeping stress ratio the same. FDG gradually decreased with crack extension and a test was manually terminated in case of crack retardation to save test duration. Subsequently, the test was repeated with increased displacements at the 
same stress ratio. This sequence was repeated several times until the maximum displacement capacity of the test machine was reached. With this test procedure, multiple delamination resistance curves were obtained, with each one representing delamination resistance equivalent to a specific fatigue pre-crack length, i.e. delamination length at which that particular fatigue test was initiated.

The presence of fibre bridging in delamination can enhance interlaminar resistance significantly. $R$-curve was therefore used in present study to quantitatively determine the critical resistance increase because of fibre bridging in FDG. However, one should note that it was difficult to directly evaluate the critical resistance $G_{I C}\left(a-a_{0}\right)$ during fatigue delamination, as fatigue load was much lower than the critical load. To address this issue, after each fatigue test, a loading-unloading procedure was added on DCB specimen to measure the critical resistance at the nonlinear point via the Modified Compliance Calibration (MCC) method recommended in the ASTM D5228-01 standard. This procedure can also provide important information for the selection of the suitable maximum and minimum displacements applied in the subsequent FDG test.

Some people may argue that load history of this test program may affect FDG behaviors. In a previous study [15], multiple fatigue delamination tests were conducted on different DCB specimens. And a uniform empirical power law relation has been successfully obtained to determine FDG with different amounts of fibre bridging. One should note that the load histories applied on different DCB specimens were not the same. If there is load-history dependence of this test procedure, one 
cannot obtain the same relation to determine FDG behaviors in different DCB specimens. In addition, it has been reported that there was a plateau state in FDG with fibre bridging [15-16]. And FDG in this state of different DCB specimens remained the same, even though the load histories were different. In our opinion, both of these can provide evidence that the effect of load history is negligible for FDG via the test procedure used in present study.

\section{FDG models}

The use of different similitude parameters in FDG study can result in various Paris type relations [5]. The basic Paris relation, see Eq.(1), has been widely used in FDG studies in composite laminates. In the previous studies via this relation [14-16], it was clearly found that the presence of fibre bridging can significantly retard fatigue crack growth. Particularly, the obtained Paris resistance curves decreased shift with crack extension, violating the requirement on similitude principle (i.e. for the same value of a similitude parameter, fatigue crack growth should be the same). To solve this problem, a modified Paris relation with a new similitude parameter, see Eq.(2), was developed and recommended to appropriately explore fibre-bridged FDG in composite laminates [23].

$$
\frac{d a}{d N}=c(\Delta \sqrt{G})^{n}=c\left[\left(\sqrt{G_{\max }}-\sqrt{G_{\min }}\right)^{2}\right]^{n}
$$

where $c$ and $n$ are two curve-fitting parameters of the Paris relation; $G_{\max }$ and $G_{\min }$ represent the maximum and minimum SERRs of a fatigue loading. They were calculated via the MCC method. The 7-point Incremental Polynominal Method, recommended in ASTM E647-00 standard, was applied to determine fatigue crack 
growth rate $d a / d N$.

$$
\frac{d a}{d N}=c^{*}\left(\Delta G_{e f f}\right)^{n^{*}}=c^{*}\left[\frac{G_{0}}{G_{I C}\left(a-a_{0}\right)} \Delta \sqrt{ } G\right]^{n^{*}}
$$

where $c^{*}$ and $n^{*}$ are two curve-fitting parameters of the modified Paris relation; $G_{0}$ represents fatigue delamination resistance with no fibre bridging; $G_{I C}\left(a-a_{0}\right)$ determines fatigue $R$-curve.

\section{Results and discussion}

\subsection{Fatigue delamination resistance}

To use the modified Paris relation Eq.(2) in determining FDG with large-scale fibre bridging, one should first evaluate the critical resistance increase in fatigue delamination, i.e. $G_{I C}\left(a-a_{0}\right)$. This parameter can be experimentally measured via the loading-unloading procedure introduced in Section 2.2 at several fatigue crack intervals. Fig.2 summarizes resistance increase during FDG in terms of $G_{I C}$ against the normalized crack extension $\left(a-a_{0}\right) / L_{p z}$. It is clear that interlaminar resistance significantly rises with crack extension from a low value to a high plateau of each thickness. This is similar to the $R$-curve usually observed in quasi-static delamination $[9,10,24]$. With crack extension, more fibre bridging can be present in the wake of a crack front and affect crack profiles, making more energy dissipation of a given crack increment. And interlaminar resistance finally can become constant as fibre bridging reaches saturation. One should note that there are two important features of the resistance increase illustrated in Fig.2. The first one is delamination resistance of composite laminates with different thicknesses remains the same at a given value of $\left(a-a_{0}\right) / L_{p z}$. As fibre bridging is the main reason for the critical resistance increase, this 
implicitly indicates the significance of fibre bridging in FDG is independent of thickness for a given normalized crack length. The second feature is the initial delamination resistance remains the same with increase of thickness. Referring to quasi-static delamination study [24], the onset crack growth was dominantly governed by the matrix property, regardless of fibre bridging. As a result, it is reasonable to have the same value of $G_{0}$.

The approach of $R$-curve was frequently employed to determine resistance increase because of fibre bridging. From the experimental results shown in Fig.2, a linear increase apparently exists between interlaminar resistance and the normalized crack extension. The linear relation proposed in literature [10], see Eq.(3), was therefore employed to quantitatively determine this increase as shown in Fig.2. This determined relationship will be used in the modified Paris relation in fatigue data analysis at the following section.

$$
G_{I C}\left(a-a_{0}\right)=\left\{\begin{array}{cl}
G_{0}+G_{b r} \frac{\left(a-a_{0}\right)}{L_{p z}} & \left(0<\frac{\left(a-a_{0}\right)}{L_{p z}} \leq 1\right) \\
G_{p l a} & \left(1<\frac{\left(a-a_{0}\right)}{L_{p z}}\right)
\end{array}\right.
$$

where $G_{p l a}$ represents resistance at the plateau state; $G_{b r}$ is the difference between $G_{p l a}$ and $G_{0} ; L_{p z}$ is the length of process zone.

\subsection{Fibre-bridged FDG in composite laminates with different thicknesses}

Incorporating with the results shown in Fig.2, all fatigue data can be interpreted via the modified Paris relation. Fig.3 provides a summary of this interpretation. And detailed Paris law representation of these fatigue data can be found in literature [25], in which the effects of fibre bridging on the Paris relation were carefully examined. 
Considering the scatters usually observed in fatigue $[3,4,22,26]$, two important features of the results shown in Fig.3 should be highlighted. The first one is the experimental data with different amounts of fibre bridging converge into a narrow band region. The second one is the experimental data from composite laminates with different thicknesses overlap each other very well. As a result, a master resistance curve can be fitted to characterize all fatigue data, as illustrated in Fig.3. Therefore, it can be concluded that both fibre bridging and composite laminate thickness have negligible effect on FDG behaviors.

The results illustrated in Fig.3 agree well with the basic requirement of similitude principle. For the same value of $\Delta G_{\text {eff, }}$ the corresponding fatigue crack growth remains the same. This means this new similitude parameter can appropriately represent the consistency or similitude in fibre-bridged FDG in composite laminates. Comparing to the results interpreted via the basic Paris relation [25], these data shown in Fig.3 highlight the importance of using an appropriate similitude parameter in correctly interpreting FDG behaviors. In the following section, both energy dissipation and damage mechanism analysis will be performed to provide physical explanations on this independence as well as to physically verify the appropriateness of the new similitude parameter.

\section{Physical interpretation of fibre-bridged FDG}

\subsection{Energy dissipation of per fatigue cycle}

FDG is an energy release process, obeying the first law of thermodynamic. Particularly, energy dissipation in FDG can be explicitly determined via Eq.(4) 
[27-28]. If there is a crack increment $d a / d N$, certain amount of energy $d U / d N$ must be available and dissipated. Knowing the energy dissipation per unit crack area $G^{*}$ (i.e. fracture toughness in FDG), the original cause $d U / d N$ can be linked to the consequence $d a / d N$. And the value of $d U / d N$ can be calculated via a derivation of the total applied energy $U$ with respect to fatigue cycle number $N$.

$$
\frac{d U}{d N}=\frac{d U}{d A} \frac{d A}{d N}=G^{*} \frac{d A}{d N}
$$

where $d A / d N=B d a / d N$ represents new crack surface generation; $B$ is the width of the sample.

All fatigue data were re-interpreted in terms of $d a / d N$ against $d U / d N$, as illustrated in Fig.4. Interestingly, the amount of energy release of a given fatigue crack growth keeps constant with fibre bridging development. This implicitly indicates there is little damage occurring in bridging fibres during FDG. As a result, the corresponding energy dissipation is negligible and most energy dissipation is actually concentrated to damage evolution around crack front. In our understanding, these bridging fibres just periodically store and release strain energy under cyclic loading, but have little contribution to permanent energy release. However, different situations exist in quasi-static delamination. The applied displacement continuously increases and can cause continuous failures in both bridging fibres and crack front. As a result, the total energy dissipation is the sum of the energy release in bridging fibres and crack front. In addition, in a FDG study by Stutz et al [17], it was reported that the magnitude of the maximum stress applied on these bridging fibres was relatively low, typically no more than 0.5 MPa. At such a low stress state, it is reasonable to believe no obvious 
fatigue damage can occur in bridging fibres. Besides fibre bridging, energy dissipation of a given fatigue crack growth is independent of thickness. And a single curve, with a coefficient of determination $R^{2}$ close to 1 , can be fitted to determine energy dissipation in fibre-bridged FDG of composite laminates with different thicknesses. It should be stressed here that the magnitude of the exponent illustrated in Fig.4 is close to unit, indicating the constant resistance $G^{*}$ during FDG with fibre bridging and thickness increase. The deviation of the exponent from unit has been well explained in a previous study by the authors [28]. Bridging fibres may be damaged or pulled out from matrix at the beginning several thousand fatigue cycles, as the applied displacement is close to the critical value. However, once crack propagates, the applied displacement becomes lower than the critical value. As a result, negligible damage related to fibre bridging can occur and all damage evolution is concentrated to crack front, leading to the same energy dissipation. The data illustrated in Fig.4 implicitly indicates the corresponding damage evolution around crack front should be the same during FDG, regardless of fibre bridging and composite laminate thickness. Detailed discussion related to the damage mechanisms will be completed in the following section via SEM observations on the fracture surfaces.

\subsection{Fractography analysis}

To provide detailed physical explanations on energy dissipation results shown in Fig.4, fractography examinations were conducted to explore the related damage mechanisms in fibre-bridged FDG of composite laminates with different thicknesses. Fig.5 
summarizes the SEM results (with the same magnification 2000X) on fatigue fracture surfaces at both short and long cracks of each thickness. The morphology for different crack lengths (i.e. different amounts of fibre bridging) of each thickness remains the same, indicating the identical damage mechanisms with fibre bridging development. In other words, fibre bridging has no obvious effect on the damage mechanisms during FDG. Particularly, fibre print is the most dominant feature located on fracture surfaces. The presence of this microscopic feature is a result of debonding between fibre and matrix around crack front. In some locations, hackles are also observed. This is a typical feature usually observed in mode II or mixed-mode I/II delamination because of shear stress. Its appearance in mode I delamination mainly attributes to local shear stress generated during fibre pullout from matrix in the wake of crack front. In comparison with the large proportion of fibre prints, the presence of hackles is relatively limited and in really small scale. Furthermore, the microscopic features remain the same with increase of laminate thickness, demonstrating the same damage mechanisms. Accordingly, one can make a conclusion that damage mechanisms in FDG remain the same with fibre bridging and thickness increase. Most damage evolution in fibre-bridged FDG is concentrated around crack front. This is the physical reason for the same energy dissipation behaviors illustrated in Fig.4.

For the identical damage mechanisms, the same crack driving force reasonably result in the same crack growth behavior. As a result, it is appropriate to use the modified Paris relation in determining fibre-bridged FDG in composite laminates. The derived results, as illustrated Fig.3, agree well with the energy dissipation results and damage 
mechanisms.

\section{Further discussion on the modified Paris relation}

According to above discussion, fibre bridging has no effect on FDG in composite laminates. Based on this, the calculated data illustrated in Fig.3 indeed quantify FDG with zero-bridging. In a recent study [29], an extrapolation method has been proposed to determine FDG with zero-bridging via extrapolating the Paris resistance curves with different amounts of fibre bridging. Fig.6 provides a comparison of the same fatigue data set $(h=5.0 \mathrm{~mm})$ interpreted via the modified Paris relation as well as calculated via the extrapolation method. As expected, FDG data calculated with different methods overlap each other very well, providing further evidence on the validity of using the modified Paris relation in fibre-bridged FDG study. In addition, Jones et al [30] provided a thorough discussion on fibre bridging in FDG and highlighted the importance of using FDG without fibre bridging in design and material characterization. The data illustrated in Fig.6 demonstrates that the use of the new fatigue model is an effective and convenient way to determine zero-bridging FDG. Furthermore, the use of the modified Paris relation in FDG study takes an advantage of resulting in a lower exponent, as compared to the basic Paris relation [25]. A smaller value of the exponent indicates less sensitivity of the prediction model. This is really important for its engineering applications. 


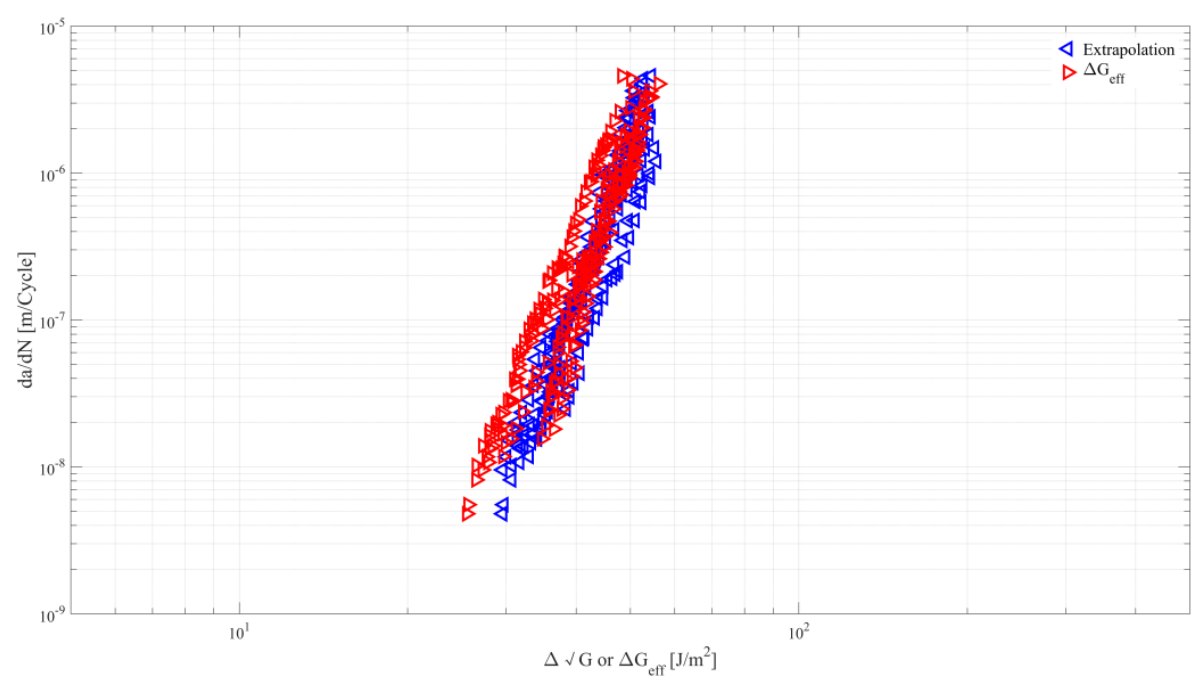

Figure 6 FDG with zero-bridging

Back to the pioneering work completed by Irwin et al [31] and Paris et al [32], crack growth was a local phenomenon. As the crack tip stress field can be governed by stress intensity factor $K$, a power law relation in terms of $d a / d N$ against the range in the governing crack tip parameter (i.e. $\Delta K$ or $K_{\max }$ ) was proposed to determine fatigue crack growth. One should bear in mind that these parameter was used to characterize crack tip stress field. However, the use of $\Delta \sqrt{ } G$ in fibre-bridged FDG violates this original intension. And one cannot make direct equivalence between $\Delta \sqrt{ } G$ and $\Delta K$. For FDG with fibre bridging, $\Delta \sqrt{ } G$ is actually a parameter to describe the total SERR range under fatigue loading. Schematically, it is the sum of SERRs in bridging fibres and around crack front. As most damage evolution and corresponding energy dissipation are concentrated around crack front, regardless of fibre bridging, the parameter which can well determine the stress field around crack front seems more appropriate in fibre-bridged FDG studies. According to above discussion and analysis, the SERR range indeed applied on crack front $\Delta G_{\text {eff }}$, rather than the total applied SERR range 
$\Delta \sqrt{ } G$, is a reasonable parameter in representing the similitude of fibre-bridged FDG. This is also in line with the original purpose of using stress intensity factor $K$ in determining fatigue crack growth.

The importance of using an appropriate similitude parameter in FDG study has been discussed in literature [5]. People alternative used $G_{\max }$ or $\Delta \sqrt{ } G$ as similitude in FDG studies in the past [2-7]. These selections were based on the description of fatigue loading, rather than physics. This shortcoming sometimes can result in misinterpreting experimental fatigue data and obscuring our understanding on FDG. Based on present and previous studies [23,27,28], physical understanding on damage evolution and corresponding energy dissipation should be conducted first for choosing a reasonable similitude parameter in fatigue data analysis. And the fatigue model based on this reasonable similitude parameter can appropriately interpret FDG behaviors and correctly enrich our understanding on FDG in composite laminates.

\section{Concluding remarks}

Thickness effects on fibre-bridged FDG in unidirectional composite laminates were investigated in present study. It was found that the significance of fibre bridging was independent of thickness at a given normalized crack extension. Experimental fatigue data were explained via a modified Paris relation. The results clearly demonstrated that both thickness and fibre bridging had no obvious effect on FDG behaviors. As a result, a single resistance curve can be obtained to determine fibre-bridged FDG in composites of different thicknesses. This finding is really important for engineering, as it can significantly reduce fatigue workload in determining FDG behaviors. 
Physical explanations on fibre-bridged FDG behaviors were provided via energy dissipation analysis and the SEM observations on fracture surfaces. The damage mechanisms remained the same with the increase of thickness and fibre bridging, causing the same energy release. Most damage evolution in fibre-bridged FDG was concentrated around crack front in terms of fibre/matrix debonding. As a result, it is reasonable to expect the same FDG behavior.

This study gives extra evidence on the importance of using an appropriate similitude parameter in FDG studies. Particularly, an appropriate similitude parameter can well interpret FDG behaviors and promote our understanding on FDG, whereas an inappropriate similitude parameter can misinterpret FDG behaviors and obscure our understanding. Furthermore, a reasonable similitude parameter can be derived by the physical understanding of the damage mechanisms and the corresponding energy dissipation behaviors.

\section{Acknowledgements}

The authors gratefully acknowledge financial support from the International Postdoctoral Exchange Fellowship Program, Harbin Institute of Technology, P.R.

China. The research work is also financially supported by the China Postdoctoral Science Foundation with grant No. 2016M601422 and Heilongjiang Postdoctoral Fund with grant No. LBH-Z16071.

\section{References}

[1] Federal Aviation Authority. Airworthiness Advisory Circular No: 20-107B. Composite Aircraft Structure. 09/08/2009 
[2] M. Hojo, K. Tanaka, C.G. Gustafson, R. Hayashi. Effect of stress ratio on near-threshold propagation of delamination fatigue cracks in unidirectional CFRP. Composites Science and Technology 1987. 29: 273-292

[3] G.B. Murri. Effect of data reduction and fiber-bridging on mode I delamination characterization of unidirectional composites. Journal of Composite Materials 2014. 48: $2413-2424$

[4] S. Stelzer, A.J. Brunner, A. Argüelles, N. Murphy, G.M. Cano, G. Pinter. Mode I delamination fatigue crack growth in unidirectional fiber reinforced composites: Results from ESIS TC4 round-robins. Engineering Fracture Mechanics 2014. 116: 92-107

[5] J.A. Pascoe, R.C. Alderliesten, R. Benedictus. Methods for the prediction of fatigue delamination growth in composites and adhesive bonds - A critical review. Engineering Fracture Mechanics 2013. 112-113: 72-96

[6] C.G. Gustafson, M. Hojo. Delamination fatigue crack growth in unidirectional Graphite/Epoxy laminates. Journal of Reinforced Plastics and Composites 1987. 6: $36-52$

[7] L. Peng, J. Zhang, L. Zhao, R. Bao, H. Yang, B. Fei. Mode I delamination growth of multidirectional composite laminates under fatigue loading. Journal of Composite Materials 2010. 45: 1077-1090

[8] D.R. Atodaria, S.K. Putatunda, P.K. Mallick. A fatigue crack growth model for random fiber composites. Journal of Composite Materials 1997. 31: 1838-1855

[9] B.F. Sorensen, T.K. Jacobsen. Large-scale bridging in composites: R-curves and 
bridging laws. Composites Part A: Applied Science and Manufacturing 1998. 29: $1443-1451$

[10] C.G. Dávila, C.A. Rose, P.P. Camanho. A procedure for superposing linear cohesive laws to represent multiple damage mechanisms in the fracture of composites. International Journal of Fracture 2009. 158: 211-223

[11] B.D. Manshadi, E. Farmand-Ashtiani, J. Botsis, A.P. Vassilopoulos. An iterative analytical/experimental study of bridging in delamination of the double cantilever beam specimen. Composites Part A: Applied Science and Manufacturing 2014. 61: $43-50$

[12] M. Hojo, S. Ochiai, T. Aoki, H. Ito. Mode I fatigue delamination for CF/PEEK laminates using maximum-energy-release-rate constant texts. Journal of the Society of Material Science Japan 1995. 44: 953-959

[13] W. Hwang, K.S. Han. Interlaminar fracture behavior and fiber bridging of glass-epoxy composite under mode I static and cyclic loadings. Journal of Composite Materials 1989. 23: 396-430

[14] L. Yao, R. Alderliesten, M. Zhao, R. Benedictus. Bridging effect on mode I fatigue delamination behavior in composite laminates. Composites Part A: Applied Science and Manufacturing 2014. 63: 103-109

[15] L. Yao, R.C. Alderliesten, R. Benedictus. The effect of fibre bridging on the Paris relation for mode I fatigue delamination growth in composites. Composite Structures 2016. 140: $125-135$

[16] L. Yao, Y. Sun, M. Zhao, R.C. Alderliesten, R. Benedictus. Stress ratio 
dependence of fibre bridging significance in mode I fatigue delamination growth of composite laminates. Composites Part A: Applied Science and Manufacturing 2017. 95: $65-74$

[17] S. Stutz, J. Cugnoni, J. Botsis. Studies of mode I delamination in monotonic and fatigue loading using FBG wavelength multiplexing and numerical analysis. Composites Science and Technology 2011. 71: 443-449

[18] R. Khan, R. Alderliesten, L. Yao, R. Benedictus. Crack closure and fibre bridging during delamination growth in carbon fibre/epoxy laminates under mode I fatigue loading. Composites Part A: Applied Science and Manufacturing 2014. 67: 201-211

[19] J. Zhang, L. Peng, L. Zhao, B. Fei. Fatigue delamination growth rates and thresholds of composite laminates under mixed mode loading. International Journal of Fatigue 2012. 40: 7-15

[20] T.K. Jacobsen, B.F. Sorensen. Mode I intra-laminar crack growth in compositesmodelling of R-curves from measured bridging laws. Composites Part A: Applied Science and Manufacturing 2001. 32: 1-11

[21] E. Farmand-Ashtiani, J. Cugnoni, J. Botsis. Specimen thickness dependence of large scale fiber bridging in mode I interlaminar fracture of carbon epoxy composite. International Journal of Solids and Structures 2015. 55: 58-65

[22] A.J. Brunner, S. Stelzer, G. Pinter, G.P. Terrasi. Cyclic fatigue delamination of carbon fiber-reinforced polymer-matrix composites: Data analysis and design considerations. International Journal of Fatigue 2016. 83: 293-299

[23] L. Yao, Y. Sun, L. Guo, M. Zhao, L. Jia, R.C. Alderliesten, R. Benedictus. A 
modified Paris relation for fatigue delamination with fibre bridging in composite laminates. Composite Structures 2017. 176: 556-564

[24] M.M. Shokrieh, M. Heidari-Rarani, M.R. Ayatollahi. Delamination R-curves as a material property of unidirectional glass/epoxy composites. Materials and Design 2012. $34: 211-218$

[25] L. Yao, Y. Sun, L. Guo, R.C. Alderliesten, R. Benedictus, M. Zhao, L. Jia. Fibre bridging effect on the Paris relation of mode I fatigue delamination in composite laminates with different thicknesses. International Journal of Fatigue 2017. 103: $196-206$

[26] M. Shahverdi, A.P. Vassilopoulos, T. Keller. Experimental investigation of R-ratio effects on fatigue crack growth of adhesively-bonded pultruded GFRP DCB joints under CA loading. Composites Part A: Applied Science and Manufacturing 2012. 43: 1689-1697

[27] L. Yao, R.C. Alderliesten, M. Zhao, R. Benedictus. Discussion on the use of the strain energy release rate for fatigue delamination characterization. Composites Part A: Applied Science and Manufacturing 2014. 66: 65-72

[28] L. Yao, R.C. Alderliesten, R. Benedictus. Interpreting the stress ratio effect on delamination growth in composite laminates using the concept of fatigue fracture toughness. Composites Part A: Applied Science and Manufacturing 2015. 78: 135-142 [29] L. Yao, J.A. Pascoe, R.C. Alderliesten. Experimental method to account for fibre bridging in mode I fatigue delamination growth data. (Under review)

[30] R. Jones, A.J. Kinloch, J. G. Michopoulos, A.J. Brunner, N. Phan. Delamination 
growth in polymer-matrix fibre composites and the use of fracture mechanics data for material characterisation and life prediction. Composite Structures 2017. 180: $316-333$

[31] G.R. Irwin. Fracture Dynamics. In Fracturing of Metals. 147-166. 1947. American Society for Metals. Cleveland.

[32] P.C. Paris, F. Erdogan. A critical analysis of crack growth propagation laws. Journal of Basic Engineering 1963. 528-533 
2018-04-16

\section{Thickness effects on fibre-bridged fatigue delamination growth in composites}

Yao, Liaojun

Elsevier

Yao L, Cui H, Alderliesten RC, Sun Y, Guo L, Thickness effects on fibre-bridged fatigue delamination growth in composites, Composites Part A: Applied Science and Manufacturing, Volume 110, July 2018, pp. 21-28 http://dx.doi.org/10.1016/j.compositesa.2018.04.015 Downloaded from Cranfield Library Services E-Repository 